\title{
Line graphs and middle graphs that are divisor graphs
}

\author{
SALAH AL-ADDASI \\ The Hashemite University \\ Faculty of Science \\ Department of Mathematics \\ Zarqa 13133 \\ JORDAN
}

\author{
HASAN AL-EZEH \\ The University of Jordan \\ Faculty of Science \\ Department of Mathematics \\ Amman 11942 \\ JORDAN
}

\author{
OMAR ABUGHNEIM \\ The University of Jordan \\ Faculty of Science \\ Department of Mathematics \\ Amman 11942 \\ JORDAN
}

Abstract: In this paper it is determined when the line graphs and the middle graphs of some classes of graphs are divisor graphs. Complete characterizations for cycles, trees, complete graphs and complete multipartite graphs whose line graphs (middle graphs) are divisor graphs are obtained. It is also shown that the line graphs and the middlegraphsofthecyclepermutationgraphsareneverdivisorgraphs.

Key-Words: Complete multipartite graph, Cycle permutation graph, Divisor graph, Line graph, Middle graph, Orientationofagraph,Tree.

Received: October 8, 2019. Revised: March 30, 2020. Accepted: April 12, 2020. Published: April 25, 2020.

\section{Introduction}

A graph $G$ is a divisor graph if the vertices of $G$ can be labeled by positive integers such that two distinct vertices $i$ and $j$ are adjacent if and only if one of the integers $i$ and $j$ divides the other. In a digraph $D$, a vertex with zero indegree is a transmitter, a vertex with zero outdegree is a receiver, while a transitive vertex $v$ has nonzero indegree and nonzero outdegree such that $(u, w) \in E(D)$ whenever $(u, v)$ and $(v, w)$ belong to $E(D)$. An orientation $D$ of a graph $G$ in which every vertex is a transmitter, a receiver, or a transitive vertex was called in [1] a divisor orientation of $G$. Divisor graphs can be interpreted by the following characterization which was proved in [6].

Lemma 1 A graph $G$ is a divisor graph if and only if $G$ has a divisor orientation.

A graph is a comparability graph if its edges can be directed so that directed adjacency becomes a transitive relation, see [13]. Thus, divisor graphs are precisely the comparability graphs.

The converse of a digraph $D$ is the digraph $D^{\prime}$ with $V(D)=V\left(D^{\prime}\right)$ and $(u, v) \in E\left(D^{\prime}\right)$ if and only if $(v, u) \in E(D)$. That is, the digraph $D^{\prime}$ is obtained from $D$ by reversing the directions of all arcs of $D$. The following result was shown in [1].

Lemma 2 If $D$ is a divisor orientation of a graph $G$, then the converse of $D$ is also a divisor orientation of G.

Some classes of graphs which are known to be divisor graphs are: Complete graphs, bipartite graphs, and complete multipartite graphs, see [6]. The graph depicted in Figure 1 was given in [6] as an example of a nondivisor graph. This graph will be referred to frequently in this paper. In [1] and [2], complete characterizations of powers of paths and powers of cycles which are divisor graphs were obtained.

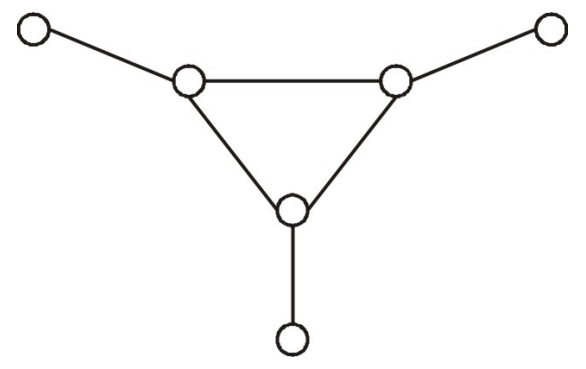

Figure 1: A graph which is not a divisor graph

The Cartesian product of two graphs $G$ and $H$ is denoted by $G \square H$. Some further properties of divisor graphs, including the following two characterizations, were shown in [3].

Lemma 3 Let $G$ and $H$ be two graphs. Then $G \square H$ is a divisor graph if and only if either both $G$ and $H$ are bipartite or at least one of them has size zero and the other is a divisor graph.

Lemma 4 Let $G$ be a block graph. Then $G$ is a divisor graph if and only if $G$ has no induced subgraph isomorphic to the graph in Figure 1.

The following two results were proved in [6]. 
Lemma 5 Every induced subgraph of a divisor graph is a divisor graph.

Lemma 6 No divisor graph contains an induced odd cycle of length greater than 3.

The middle graph $M(G)$ of a graph $G$ has vertex set $V(G) \cup E(G)$ where two vertices of $M(G)$ are adjacent if either they are incident edges in $G$ or one of them is an edge of $G$ and the other is a vertex incident to it in $G$, see [12].

The join of two disjoint graphs $G$ and $H$, denoted by $G+H$, is obtained from $G \cup H$ by adding all edges $x y$ with $x \in V(G)$ and $y \in V(H)$. The set of neighbors of a vertex $v$ in a graph is denoted by $N(v)$. For undefined notions, the reader is referred to [5]. In this paper, all graphs we consider are connected and contain neither loops nor multiple edges.

\section{Cycles and trees}

Every cycle is isomorphic to its line graph. Since even cycles are bipartite graphs and $C_{3}$ is a complete graph, the following result follows immediately by Lemma 6 .

Proposition $7 L\left(C_{n}\right)$ is a divisor graph if and only if either $n$ is even or $n=3$.

Now we show that the graph depicted in Figure 2 is a forbidden subgraph in the graph $G$ for $L(G)$ to be a divisor graph.

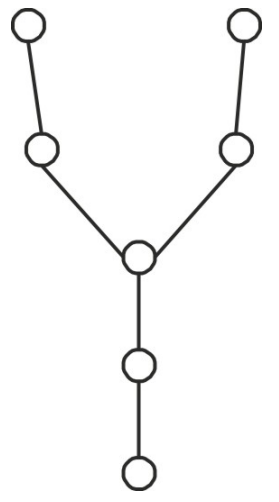

Figure 2: A forbidden subgraph in a graph $G$ for $L(G)$ to be a divisor graph

Proposition 8 If $G$ is a graph containing a subgraph isomorphic to the graph in Figure 2, then $L(G)$ is not a divisor graph.

Proof: Let $G$ be a graph containing a subgraph $H$ which is isomorphic to the graph in Figure 2. Then $L(H)$ is an induced subgraph of $L(G)$. But $L(H)$ is isomorphic to the graph in Figure 1, which is not a divisor graph. Therefore, by Lemma $5, L(G)$ is not a divisor graph.

A caterpillar is a tree containing a path with the property that every vertex is at distance at most one from this path, see [7]. It is obvious that a caterpillar is a tree that contains no subgraph isomorphic to that in Figure 2. Now, we will characterize the trees whose line graphs are divisor graphs. It is well known that the line graphs of trees are block graphs, see [9].

Theorem 9 Let $T$ be a nontrivial tree. Then $L(T)$ is a divisor graph if and only if $T$ is a caterpillar.

Proof: Since $T$ is a tree, we have $L(T)$ is a block graph. Note that the line graph of the graph in Figure 2 is just the graph in Figure 1. Assume that $L(T)$ is a divisor graph. Then by Lemma 4 we get that $L(T)$ does not contain the graph in Figure 1 as an induced subgraph. Thus $T$ does not contain a subgraph isomorphic to that in Figure 2, and hence $T$ is a caterpillar.

Conversely, assume that $T$ is a caterpillar. Then $T$ does not contain the graph in Figure 2 as a subgraph. Thus the graph $L(T)$ does not contain an induced subgraph isomorphic to that in Figure 1. Therefore by Lemma 4 we get that $L(T)$ is a divisor graph.

It is clear that the middle graph $M(G)$ of a graph $G$ contains $L(G)$ as an induced subgraph. Therefore, by Lemma 5, we have the following result.

Proposition 10 For any graph $G$, if $L(G)$ is not a divisor graph, then $M(G)$ is also not a divisor graph.

Recall that for $n \geq 3$, the $n$-sun is the graph obtained from the complete graph on the $n$ vertices $u_{1}, u_{2}, \cdots, u_{n}$ by adding new $n$ vertices $w_{1}, w_{2}, \cdots, w_{n}$ and adding the edges $w_{i} u_{i}, w_{i} u_{i+1}$ (modulo $n$ ), for $i=1,2, \cdots, n$. Before turning to the middle graphs of cycles, we consider the 3 -sun.

\section{Lemma 11 The 3-sun is not a divisor graph.}

Proof: Let $G$ be the 3-sun with $V(G)=$ $\left\{u_{1}, u_{2}, u_{3}, w_{1}, w_{2}, w_{3}\right\}$, where $\left\{u_{1}, u_{2}, u_{3}\right\}$ induces $K_{3}$ and $w_{i}$ is adjacent to both $u_{i}$ and $u_{i+1}$ (modulo 3 ), for $i=1,2,3$. Assume to the contrary that $G$ is a divisor graph. Then, by Lemma $1, G$ has a divisor orientation $D$. In view of Lemma 2 , suppose that $\left(w_{2}, u_{2}\right) \in E(D)$. Then, since $w_{2} u_{1}, w_{2} w_{1} \notin E(G)$, we must have $\left(u_{1}, u_{2}\right),\left(w_{1}, u_{2}\right) \in E(D)$. Then we get $\left(u_{3}, u_{2}\right) \in E(D)$ because $w_{1} u_{3} \notin E(G)$. Now distinguish two cases:

Case (1) $\left(w_{1}, u_{1}\right) \in E(D)$. 
Then, since $w_{1} w_{3} \notin E(G)$, we must have $\left(w_{3}, u_{1}\right) \in E(D)$. Thus we have $\left(w_{3}, u_{1}\right),\left(u_{1}, u_{2}\right) \in$ $E(D)$ while $\left(w_{3}, u_{2}\right) \notin E(D)$, a contradiction.

Case (2) $\left(u_{1}, w_{1}\right) \in E(D)$.

Then, since $w_{3} w_{1}, u_{3} w_{1} \notin E(G)$, we must have $\left(u_{1}, w_{3}\right),\left(u_{1}, u_{3}\right) \in E(D)$. Then we have $\left(w_{2}, u_{3}\right) \in$ $E(D)$ because $u_{1} w_{2} \notin E(G)$. But $w_{2} w_{3} \notin E(G)$, so we must have $\left(w_{3}, u_{3}\right) \in E(D)$. Thus we have $\left(w_{3}, u_{3}\right),\left(u_{3}, u_{2}\right) \in E(D)$ while $\left(w_{3}, u_{2}\right) \notin E(D)$, a contradiction.

Therefore $G$ is not a divisor graph.

The following lemma assures that the middle graph of a graph containing a triangle or an induced 3 -star is not a divisor graph.

Lemma 12 If the middle graph $M(G)$ of a graph $G$ is a divisor graph, then $G$ is triangle-free and contains no induced $K_{1,3}$.

Proof: Assume that $G$ contains a triangle 1231 . Then the set $\{1,2,3,12,13,23\}$ induces in $M(G)$ a 3 -sun. Therefore, by Lemma 5, $M(G)$ is not a divisor graph. Next, assume that $K_{1,3}$ is an induced subgraph of $G$. Let $V\left(K_{1,3}\right)=\{x, 1,2,3\}$ and $E\left(K_{1,3}\right)=$ $\{1 x, 2 x, 3 x\}$. Then the set $\{1,2,3,1 x, 2 x, 3 x\}$ induces in $M(G)$ a subgraph isomorphic to that in Figure 1. Thus, by Lemma $5, M(G)$ is not a divisor graph.

Now we can determine the values of $n$ for which $M\left(C_{n}\right)$ is a divisor graph.

Theorem $13 M\left(C_{n}\right)$ is a divisor graph if and only if $n$ is even .

Proof: For odd $n>3$, the middle graph $M\left(C_{n}\right)$ is not a divisor graph according to Proposition 7 and Proposition 10. The middle graph $M\left(C_{3}\right)$ is not a divisor graph, by Lemma 12. Let $C_{n}$ be the cycle $x_{1} x_{2} \ldots x_{n} x_{1}$ where $n$ is even, and for $1 \leq i \leq n$ let $e_{i}$ be the edge $x_{i} x_{i+1}$, where the index is computed modulo $n$. Define the orientation $D$ of $M\left(C_{n}\right)$ as follows: For odd $i$, let $e_{i}$ be a receiver, and for even $i$, let $e_{i}$ be a transmitter. Note that $e_{i}$ and $e_{i-1}$ are the only neighbors of $x_{i}$ in $M\left(C_{n}\right)$, where the index is computed modulo $n$. Now, if $i$ is odd, then $\left(e_{i-1}, x_{i}\right),\left(x_{i}, e_{i}\right)$ and $\left(e_{i-1}, e_{i}\right)$ belong to $E(D)$. And if $i$ is even, then $\left(e_{i}, x_{i}\right),\left(x_{i}, e_{i-1}\right)$ and $\left(e_{i}, e_{i-1}\right)$ belong to $E(D)$. Thus for every $i$, the vertex $x_{i}$ is a transitive vertex in $D$. Then $D$ is a divisor orientation of $M\left(C_{n}\right)$. Therefore $M\left(C_{n}\right)$ is a divisor graph.

Note that the paths are precisely the $K_{1,3}$-free trees.

Theorem 14 For any tree $T$, the middle graph $M(T)$ is a divisor graph if and only if $T$ is a path.
Proof: Let $T$ be a tree which is not a path. Then $T$ contains a $K_{1,3}$. Thus, by Lemma $12, M(T)$ is not a divisor graph. So, assume that $T$ is the path $P_{n}$. The graphs $M\left(P_{1}\right)=K_{1}$ (complete) and $M\left(P_{2}\right)=P_{3}$ (bipartite) are divisor graphs. For $n \geq 3$, let $P_{n}$ be the path $12 \ldots n$ and let $e_{i}(1 \leq i \leq n-1)$ be the edge $i(i+$ $1)$. Define the orientation $D$ of $M\left(P_{n}\right)$ as follows: Let $e_{i}$ be a transmitter when $i$ is even, and a receiver when $i$ is odd. Then the vertex 1 is a transmitter in $D$, and the vertex $n$ is a transmitter when $n$ is even and a receiver when $n$ is odd. For each $2 \leq i \leq n-1$, the vertex $i$ has exactly two neighbors $e_{i-1}$ and $e_{i}$. When $i$ is even we have $\left(e_{i}, i\right),\left(i, e_{i-1}\right)$ and $\left(e_{i}, e_{i-1}\right)$ belong to $E(D)$, while when $i$ is odd we have $\left(e_{i-1}, i\right),\left(i, e_{i}\right)$ and $\left(e_{i-1}, e_{i}\right)$ belong to $E(D)$. Thus for $2 \leq i \leq$ $n-1$, the vertex $i$ is a transitive vertex in $D$. Then $D$ is a divisor orientation of $M\left(P_{n}\right)$. Therefore $M\left(P_{n}\right)$ is a divisor graph.

\section{Complete and complete multipar- tite graphs}

The vertex set of the complete $k$-partite graph $K_{r_{1}, r_{2}, \cdots, r_{k}}$ consists of $k$ disjoint sets having cardinalities $r_{1}, r_{2}, \cdots, r_{k}$ and each vertex is adjacent to all others except those in the same set. If the $k$ sets have the same cardinality $r$, then the complete $k$-partite graph $K_{r, r, \cdots, r}$ is denoted by $K_{k(r)}$. The graph $K_{k(2)}$ is called the cocktail graph or the $k$-dimensional octahedral graph, see [4].

The line graphs of complete graphs are divisor graphs only for small complete graphs, as the following result says.

Theorem 15 For $n>1$, the line graph $L\left(K_{n}\right)$ is a divisor graph if and only if $n<5$.

Proof: For $n=2,3$, the graph $L\left(K_{n}\right)$ is complete, and hence $L\left(K_{n}\right)$ is a divisor graph. The graph $L\left(K_{4}\right)$ is the octahedron graph $K_{3(2)}$ (multipartite). Thus $L\left(K_{4}\right)$ is a divisor graph. So, suppose that $n \geq 5$ and let $V\left(K_{n}\right)=\{1,2, \ldots, n\}$. Then the set $\{12,13,14,52,53,54\}$ of vertices induces in $L\left(K_{n}\right)$ a subgraph isomorphic to $K_{2} \square K_{3}$. But, by Lemma 3, $K_{2} \square K_{3}$ is not a divisor graph. Therefore, by Lemma $5, L\left(K_{n}\right)$ is not a divisor graph.

The graphs $M\left(K_{1}\right)$ and $M\left(K_{2}\right)$ are divisor graphs. Thus, in view of Proposition 10 and Lemma 12 , we have the following result.

Theorem $16 M\left(K_{n}\right)$ is a divisor graph if and only if $n<3$. 
Now we will show that $L(G)$ and $M(G)$ cannot be divisor graphs whenever $G$ contains $K_{2,3}$. This result will be needed in the rest of this section.

Proposition 17 Let $G$ be a graph containing $K_{2,3}$. Then $L(G)$ and $M(G)$ are not divisor graphs.

Proof: Clearly, $L\left(K_{2,3}\right)=K_{2} \square K_{3}$ is an induced subgraph of $L(G)$. Then, by Lemma 3 and Lemma 5, $L(G)$ is not a divisor graph. Thus, by Proposition 10, also $M(G)$ is not a divisor graph.

Before turning to the line graphs of complete multipartite graphs, let us settle the case of complete bipartite graphs.

Lemma $18 L\left(K_{m, n}\right)$ is a divisor graph if and only if either $\min \{m, n\}=1$ or $m=n=2$.

Proof: The line graph $L\left(K_{1, n}\right)$ is the complete graph $K_{n}$, which is a divisor graph. The line graph $L\left(K_{2,2}\right)$ is just $C_{4}$, which is a divisor graph. Suppose that $\min \{m, n\} \geq 2$ and $\max \{m, n\} \geq 3$. Then $K_{m, n}$ contains a subgraph isomorphic to $K_{2,3}$. Thus, by Proposition 17, $L\left(K_{m, n}\right)$ is not a divisor graph.

Now we are in a position to characterize those complete $k$-partite graphs whose line graphs are divisor graphs. They are namely $K_{1, n}(n \geq 1), K_{2,2}$, $K_{2,1,1}, K_{1,1,1}$ and $K_{1,1,1,1}$.

Theorem 19 The line graph of the complete k-partite graph $K_{r_{1}, r_{2}, \cdots, r_{k}}$ is a divisor graph if and only if one of the following conditions holds:

(1) $k \leq 4$ and $r_{i}=1$ for every $i$.

(2) $k=2$ and either $r_{1}=r_{2}=2$ or $\min \left\{r_{1}, r_{2}\right\}=1$.

(3) $k=3$ and $r_{t}=2$ for some $t$ with $r_{i}=1$ for every $i \neq t$.

Proof: Let $G=K_{r_{1}, r_{2}, \cdots, r_{k}}$. If $r_{i}=1$ for every $i$, then $G=K_{k}$. Thus, by Theorem $15, L(G)$ is a divisor graph if and only if $k \leq 4$. If $k=2$, then the result follows by Lemma 18. So suppose that $k \geq 3$ and there exists $i$ such that $r_{i} \geq 2$. Without loss of generality, we can assume that $i=1$. Let $U_{1}, U_{2}, \cdots, U_{k}$ be the parts of the complete $k$-partite graph $G$ with cardinalities $r_{1}, r_{2}, \cdots, r_{k}$, respectively. Consider the following three cases:

Case (1) $k \geq 4$.

Let $S=\left\{a, b, x_{2}, x_{3}, x_{4}\right\}$, where $a, b \in U_{1}$ and $x_{i} \in U_{i}$ for $i=2,3,4$. Then the subgraph of $G$ induced by $S$ contains a subgraph isomorphic to $K_{2,3}$. Therefore, by Proposition 17, $L(G)$ is not a divisor graph.

Case (2) $k=3$ and $r_{1} \geq 3$.
Let $S=\left\{a, b, c, x_{2}, x_{3}\right\}$, where $a, b, c \in U_{1}$ and $x_{i} \in U_{i}$ for $i=2,3$. Then the subgraph of $G$ induced by $S$ contains a subgraph isomorphic to $K_{2,3}$. Therefore, by Proposition 17, $L(G)$ is not a divisor graph.

Case (3) $k=3$ and $r_{1}=2$.

If at least one of $U_{2}, U_{3}$ has more than one element. Then the set $S=\{a, b, x, y, z\}$, where $a, b \in U_{1}$ and $x, y, z \in U_{2} \cup U_{3}$, induces in $G$ a subgraph containing $K_{2,3}$. Therefore, by Proposition $17, L(G)$ is not a divisor graph. So, suppose that $\left|U_{2}\right|=\left|U_{3}\right|=1$. Then $G=K_{2}+2 K_{1}$, and hence $L(G)=W_{1,4}$. But $W_{1,4}$ has a divisor orientation in which the vertex of degree 4 in $W_{1,4}$ is made a transmitter and two other nonadjacent vertices are made receivers. Therefore $L(G)$ is a divisor graph.

The following result shows that among all multipartite graphs, the middle graphs of only three bipartite graphs (namely: $K_{1,1}, K_{1,2}$ and $K_{2,2}$ ) are divisor graphs.

Theorem 20 The middle graph $M\left(K_{r_{1}, r_{2}, \ldots, r_{k}}\right)$ is a divisor graph if and only if $k=2$ and $\max \left\{r_{1}, r_{2}\right\} \leq$ 2 .

Proof: In view of Proposition 10 and Theorem 19, we need only to study the three cases in the statement of Theorem 19. When $k \leq 4$ and $r_{i}=1$ for every $i$, the multipartite graph $K_{r_{1}, r_{2}, \ldots, r_{k}}$ equals $K_{k}$. Thus, by Theorem 16, only $M\left(K_{1,1}\right)$ is a divisor graph. Since $K_{2,2}=C_{4}$, we have $M\left(K_{2,2}\right)$ is a divisor graph by Theorem 13 . For every $n \geq 3$, we have $M\left(K_{1, n}\right)$ is not a divisor graph according to Lemma 12 . The middle graph $M\left(K_{1,2}\right)$ has a divisor orientation in which one of the edges of $K_{1,2}$ is taken as a transmitter while the other edge is a receiver, hence $M\left(K_{1,2}\right)$ is a divisor graph. Finally, $M\left(K_{2,1,1}\right)$ is not a divisor graph, by Lemma 12 .

\section{Cycle permutation graphs}

For $n \geq 3$, a cycle permutation graph $P_{\alpha}\left(C_{n}\right)$ consists of two copies of the $n$-cycle $12 \cdots n 1$ such that every vertex $i$ of one copy is adjacent to the vertex $\alpha(i)$ in the other copy, where $\alpha$ is a permutation on $V\left(C_{n}\right)$, see [10]. The Petersen graph is the cycle permutation graph $P_{\alpha}\left(C_{5}\right)$ where $\alpha$ is the permutation $\left(\begin{array}{lllll}1 & 2 & 3 & 4 & 5 \\ 2 & 4 & 1 & 3 & 5\end{array}\right)$.

The following result shows that the line graph (and hence the middle graph) of a cycle permutation graph is never a divisor graph.

Theorem 21 For $n \geq 3$, the graphs $L\left(P_{\alpha}\left(C_{n}\right)\right)$ and $M\left(P_{\alpha}\left(C_{n}\right)\right)$ are not divisor graphs. 
Proof: Consider the following three cases:

Case (1) $n \geq 5$.

Let $\alpha(i) \in N(\alpha(3))-\{3\}$ in the graph $P_{\alpha}\left(C_{n}\right)$. Then the set $\{12,23,34,45,3 \alpha(3), \alpha(3) \alpha(i)\}$ of vertices induces in $L\left(P_{\alpha}\left(C_{n}\right)\right)$ a subgraph isomorphic to the graph in Figure 1. Therefore, by Lemma 5, $L\left(P_{\alpha}\left(C_{n}\right)\right)$ is not a divisor graph.

Case (2) $n=4$.

Let $\alpha(i) \in N(\alpha(3))-\{3\}$ in the graph $P_{\alpha}\left(C_{n}\right)$ such that $\alpha(i) \neq \alpha(4)$. Then the set $\{12,23,34,3 \alpha(3), 4 \alpha(4), \alpha(3) \alpha(i)\}$ of vertices induces in $L\left(P_{\alpha}\left(C_{n}\right)\right)$ a subgraph isomorphic to the graph in Figure 1. Therefore, by Lemma 5, $L\left(P_{\alpha}\left(C_{n}\right)\right)$ is not a divisor graph.

Case (3) $n=3$.

Note that $P_{\alpha}\left(C_{3}\right)$ is just $K_{2} \square K_{3}$ for any $\alpha$. Let $V\left(K_{2}\right)=\{a, b\}$ and $V\left(K_{3}\right)=\{1,2,3\}$. Then the six vertices $(a, 1)(a, 2), \quad(a, 1)(a, 3)$, $(a, 2)(a, 3),(a, 1)(b, 1),(a, 2)(b, 2),(a, 3)(b, 3)$ induce in $L\left(P_{\alpha}\left(C_{3}\right)\right)$ a 3 -sun. Therefore, by Lemma 11 and Lemma 5, $L\left(P_{\alpha}\left(C_{3}\right)\right)$ is not a divisor graph. Thus, by Proposition 10, also $M\left(P_{\alpha}\left(C_{n}\right)\right)$ is not a divisor graph for any $n \geq 3$.

\section{Conclusion}

Note that all trees are divisor graphs. But in view of Theorem $9, L(T)$ is a divisor graph for some trees $T$, and $L(T)$ is not a divisor graph for other trees $T$. On the other hand, the graph of Figure 1 as well as its line graph (which is the 3 -sun) are not divisor graphs. It would be interesting to determine whether the condition that $G$ is a divisor graph is necessary for $L(G)$ to be a divisor graph.

Graph theory is fundamental in computer science. For using graph theory in a modern way attractive to students, the reader is referred to [11]. Graphs are essentially used in modelling communication networks, see for example [8].

In this paper we have obtained some characterizations concerning divisor graphs. As mentioned in the introduction, divisor graphs are also known as comparability graphs. It is shown in [14] that in many media applications the stream interference graphs are comparability graphs or decomposable into comparability graphs.

Acknowledgments: The authors thank the referees for their valuable comments.

\section{References:}

[1] S. Al-Addasi, O.A. AbuGhneim, and H. AlEzeh, Divisor orientations of powers of paths and powers of cycles, Ars Combin. 94, 2010, pp. 371-380.

[2] S. Al-Addasi, O.A. AbuGhneim, and H. AlEzeh, Characterizing powers of cycles that are divisor graphs, Ars Combin. 97 A, 2010, pp. 447-451.

[3] S. Al-Addasi, O.A. AbuGhneim, and H. AlEzeh, Further new properties of divisor graphs, J. Combin. Math. Combin. Comput. 81, 2012, pp. 261-272.

[4] L. Beineke, R. Wilson and P. Cameron, Introduction. In: L. Beineke and R. Wilson (Eds.), P. Cameron (Academic Consultant), Topics in Algebraic Graph Theory, Cambridge University Press, New York, 2005, pp. 1-29.

[5] F. Buckley and M. Lewinter, A Friendly Introduction to Graph Theory, Prentice Hall, New Jersey 2003.

[6] G. Chartrand, R. Muntean, V. Saenpholphat, and P. Zhang, Which graphs are divisor graphs?, Congr. Numer. 151, 2001, pp. 189-200.

[7] A.M. Dean and J.P. Hutchinson, Visibility graphs. In: J.L. Gross, J. Yellen and P. Zhang (Eds.), Handbook of Graph Theory, CRC Press LLC, Boca Raton, 2nd ed. 2014, pp. 1348-1367.

[8] S. Deswal and A. Singhrova, Applications of graph theory in communication networks, IJAIEM Vol. 1, 2, 2012, pp. 66-70.

[9] F. Harary, Graph Theory, 3rd ed., AddisonWesley, Massachusetts, 1972.

[10] J.H. Kwak and J. Lee, Enumeration of graph coverings, surface branched coverings and related group theory. In: S. Hong, J.H. Kwak, K.H. Kim, and F.W. Roush (Eds.), Combinatorial \& Computational Mathematics: Present and Future, World Scientific, Singapore, 2001, pp. 97161.

[11] E. Milkova and A. Sevcikova, Deeper insight into graph theory using multimedia applications, International Journal of Circuits, Systems and Signal Processing Vol. 10, 2016, pp. 448-454.

[12] K. Sutha, K. Thirusangu and S. Bala, Some graph labelings on middle graph of extended duplicate graph of a path, Annals of Pure and Applied Mathematics Vol. 8, No. 2, 2014, pp. 169174.

[13] A. Tucker, Perfect graphs. In: J.L. Gross and J. Yellen (Eds.), Handbook of Graph Theory, CRC Press LLC, Boca Raton, 2004, pp. 431-444.

[14] X. Yang, L. Wang, J. Xue and Q. Wu, Comparability graph coloring for optimizing utilization of software-managed stream register files for stream processors, ACM Trans. Archit. Code Optim. 9, 1, Article 5, 2012, 30 pages. 